\title{
Exact distribution of a modified Behrens-Fisher statistic
}

DOI:

10.1080/03610918.2016.1213283

\section{Document Version}

Accepted author manuscript

Link to publication record in Manchester Research Explorer

\section{Citation for published version (APA):}

Nadarajah, S., \& Li, R. (2016). Exact distribution of a modified Behrens-Fisher statistic. Communications in Statistics: Simulation and Computation, 1-20. https://doi.org/10.1080/03610918.2016.1213283

\section{Published in:}

Communications in Statistics: Simulation and Computation

\section{Citing this paper}

Please note that where the full-text provided on Manchester Research Explorer is the Author Accepted Manuscript or Proof version this may differ from the final Published version. If citing, it is advised that you check and use the publisher's definitive version.

\section{General rights}

Copyright and moral rights for the publications made accessible in the Research Explorer are retained by the authors and/or other copyright owners and it is a condition of accessing publications that users recognise and abide by the legal requirements associated with these rights.

\section{Takedown policy}

If you believe that this document breaches copyright please refer to the University of Manchester's Takedown Procedures [http://man.ac.uk/04Y6Bo] or contact uml.scholarlycommunications@manchester.ac.uk providing relevant details, so we can investigate your claim.

\section{OPEN ACCESS}




\title{
Exact distribution of a modified Behrens-Fisher statistic
}

\author{
Saralees Nadarajah and Rui Li \\ School of Mathematics, University of Manchester, UK
}

June 15, 2017

\begin{abstract}
The exact distribution of a modified Behrens-Fisher statistic is derived. The distribution function is mostly elementary and is simpler than the exact distribution derived by Nel et al. [Communications in Statistics - Theory and Methods, 19, 1990, 279-298]. Its practical use (including computational efficiency and computational convenience) is discussed.
\end{abstract}

Keywords: Behrens-Fisher statistic; Hypergeometric functions; Student's $t$ distribution.

\section{Introduction}

Suppose $x_{1}, x_{2}, \ldots, x_{m}$ is a random sample from a normal population with mean $\mu_{X}$ and variance $\sigma_{X}^{2}$. Suppose $y_{1}, y_{2}, \ldots, y_{n}$ is another random sample from a normal population with mean $\mu_{Y}$ and variance $\sigma_{Y}^{2}$. Suppose too that the random samples are independent. If the variances are unknown and unequal then the test for equality of means is based on the statistic

$$
\frac{\bar{x}-\bar{y}}{\sqrt{\frac{s_{X}^{2}}{m}+\frac{s_{Y}^{2}}{n}}}=\mathcal{T}
$$

say, where

$$
\begin{aligned}
& \bar{x}=\frac{1}{m} \sum_{i=1}^{m} x_{i}, \quad \bar{y}=\frac{1}{n} \sum_{i=1}^{n} y_{i}, \\
& s_{X}^{2}=\frac{1}{m-1} \sum_{i=1}^{m}\left(x_{i}-\bar{x}\right)^{2}, \quad s_{Y}^{2}=\frac{1}{n-1} \sum_{i=1}^{n}\left(y_{i}-\bar{y}\right)^{2} .
\end{aligned}
$$

(1) is known as the Behrens-Fisher statistic (Fisher, 1935). Commonly its distribution is approximated by a Student's $t$ random variable (Welch, 1938) with degree of freedom

$$
\nu=\frac{\left(\frac{s_{X}^{2}}{m}+\frac{s_{Y}^{2}}{n}\right)^{2}}{\frac{1}{m-1}\left(\frac{s_{X}^{2}}{m}\right)^{2}+\frac{1}{n-1}\left(\frac{s_{Y}^{2}}{n}\right)^{2}} .
$$

The resulting test is known as the two sample $t$ test. Many other approximations to the distribution of (1) have since been proposed. We refer the readers to Chapters 28 and 30 of Johnson et al. (1995) for an excellent review. See also Kim and Cohen (1998). 
There has been little work on deriving the exact distribution of (1). The only work known to us is that due to Nel et al. (1990). Under the hypothesis $\mu_{X}=\mu_{Y}$, Nel et al. (1990) showed that the probability density function (pdf) of $\mathcal{T}$ can be expressed as

$$
\begin{aligned}
f_{\mathcal{T}}(t)= & \frac{\Gamma\left(\frac{m+n+1}{2}\right)}{\sqrt{\pi} \Gamma\left(\frac{m+n}{2}\right) g_{X}^{m / 2} g_{Y}^{n / 2} \sqrt{\phi^{2}}}\left(\frac{1}{g_{Y}}+\frac{t^{2}}{\phi^{2}}\right)^{-\frac{m+n+1}{2}} \\
& \cdot_{2} F_{1}\left(\frac{m}{2}, \frac{m+n+1}{2} ; \frac{m+n}{2} ;\left(\frac{1}{g_{Y}}-\frac{1}{g_{X}}\right)\left(\frac{1}{g_{Y}}+\frac{t^{2}}{\phi^{2}}\right)^{-1}\right),
\end{aligned}
$$

where $g_{X}=\sigma_{X}^{2} /[m(m-1)], g_{Y}=\sigma_{Y}^{2} /[n(n-1)], \phi^{2}=m^{-1} \sigma_{X}^{2}+n^{-1} \sigma_{Y}^{2}$ and ${ }_{2} F_{1}(a, b ; c ; x)$ denotes the Gauss hypergeometric function defined by

$$
{ }_{2} F_{1}(a, b ; c ; x)=\sum_{k=0}^{\infty} \frac{(a)_{k}(b)_{k}}{(c)_{k}} \frac{x^{k}}{k !}
$$

where $(e)_{k}=e(e+1) \cdots(e+k-1)$ denotes the ascending factorial. Nel et al. (1990) did not give expressions for the cumulative distribution function (cdf) of $\mathcal{T}$.

The Gauss hypergeometric function in (3) is a special function and there are not many computer programs for computing it. Even some of the programs for computing the Gauss hypergeometric function are prone to round off errors, for example, hypergeom in the R software ( $\mathrm{R}$ Development Core Team, 2015). Besides, many authors have stated that (3) is "computationally intractable". For example, Krishnamoorthy and Yu (2004) stated that (3) is "computationally intractable ... is of no use for practical applications". For multivariate Behrens-Fisher problem, Kakizawa and Iwashita (2008) stated that (3) is "quite complicated and intractable from a computational point of view".

The aim of this paper is to derive the exact distribution of (1) in mostly elementary forms. In fact, the derived forms for the pdf and cdf are all elementary except when both $m$ and $n$ are even numbers. Elementary forms can be computed accurately on any platform, even using a pocket calculator. Hence, they are computationally more convenient than (3).

In passing, we would like to point out that even some of the known approximations for (1) are not elementary. For example, the Student's $t$ approximation's distribution function involves the incomplete beta function ratio, a special function, for all $m$ and $n$.

Consider the modified form of (1) given by

$$
\frac{\left[(\bar{x}-\bar{y})-\left(\mu_{X}-\mu_{Y}\right)\right]^{2}}{\frac{s_{X}^{2}}{m}+\frac{s_{Y}^{2}}{n}}=T
$$

say. Note that (1) and (4) are equivalent at least when $\mu_{X}=\mu_{Y}$. Using the facts

$$
\begin{aligned}
& \bar{x} \sim N\left(\mu_{X}, \frac{\sigma_{X}^{2}}{m}\right), \quad \bar{y} \sim N\left(\mu_{Y}, \frac{\sigma_{Y}^{2}}{n}\right), \\
& \frac{(m-1) s_{X}^{2}}{\sigma_{X}^{2}} \sim \chi_{m-1}^{2}, \quad \frac{(n-1) s_{Y}^{2}}{\sigma_{Y}^{2}} \sim \chi_{n-1}^{2},
\end{aligned}
$$

we can rewrite (4) as

$$
T=\frac{U}{V+W},
$$

where $U \sim \chi_{1}^{2}, V \sim \Gamma(a, b), W \sim \Gamma(c, d)$ are independent random variables and

$$
\begin{aligned}
& a=\frac{m-1}{2}, \quad c=\frac{n-1}{2}, \\
& b=\frac{(m-1)\left(n \sigma_{X}^{2}+m \sigma_{Y}^{2}\right)}{2 n \sigma_{X}^{2}}, \quad d=\frac{(n-1)\left(n \sigma_{X}^{2}+m \sigma_{Y}^{2}\right)}{2 m \sigma_{Y}^{2}} .
\end{aligned}
$$


Here, $\Gamma(a, b)$ denotes a gamma random variable with shape parameter $a$ and rate parameter $b$.

Exact expressions for the pdf and cdf of $T$ in (5) are given in Section 2. Of the expressions given there, the ones that are new and original are: the cdf of $T$ in the general case; the pdf and cdf of $T$ when $b=d$; the cdf of $T$ when $m$ and $n$ are odd; the cdf of $T$ when $m$ is odd and $n$ is even; the cdf of $T$ when $m$ is even and $n$ is odd; the cdf of $T$ when $m$ and $n$ are even; the pdf of $T$ when $m$ is odd; the pdf of $T$ when $n$ is odd; the pdf of $T$ when $m$ and $n$ are even. All of the proofs are given in Appendix A.

The practical use and efficiency of the newly derived expressions are discussed in Section 3. The discussion centers on four aspects: i) comparison of the newly derived pdfs and the Student's $t$ pdf with degree of freedom (2); ii) comparison of the newly derived cdfs and the Student's $t$ cdf with degree of freedom (2); iii) comparison of power functions corresponding to the newly derived expressions and the Student's $t$ approximation with degree of freedom (2); iv) comparison of computational times corresponding to the newly derived expressions and (3).

The expressions given in Section 2 are mostly elementary. One case giving non-elementary expressions is when both $m$ and $n$ are even integers. In this case, expressions involve the error function, modified Bessel function of the first kind and elliptical integrals. The derivations in all cases make use of the error function and the confluent and Gauss hypergeometric functions. The derivations also make use of some new integral identities hitherto unknown. These new identities are stated and proved in Appendix B.

\section{Main results}

The main results are explicit expressions for the cdf and pdf of $T$ in (5). All of the expressions are new and original except for those given by (7) and (8). The latter expressions are due to Nel et al. (1990). The practical use and efficiency of most of the expressions are illustrated in Section 3.

Throughout, we suppose $U \sim \chi_{1}^{2}, V \sim \Gamma(a, b)$ and $W \sim \Gamma(c, d)$ are independent random variables, where $a>0, b>0, c>0$ and $d>0$. The expressions are grouped as follows: the general case where there are no restrictions on $a>0, b>0, c>0$ and $d>0$; the case $b=d>0$ but no restrictions on $a>0$ and $c>0$; the case $m>1$ is an odd integer; the case $n>1$ is an odd integer; the case $m>1$ and $n>1$ are even integers. We shall see that all of the derived expressions are elementary except for the general case and the case $m>1$ and $n>1$ are even integers.

\subsection{General case}

Suppose $a>0, b>0, c>0$ and $d>0$ are real numbers. Then the cdf of $T$ can be expressed as

$$
F_{T}(t)=\frac{b^{a} d^{c}}{\Gamma(a+c)} \int_{0}^{\infty} z^{a+c-1} \exp (-d z) \operatorname{erf}(\sqrt{t z / 2}){ }_{1} F_{1}(a ; a+c ;-(b-d) z) d z
$$

for $t>0$, where $\operatorname{erf}(x)$ denotes the error function defined by

$$
\operatorname{erf}(x)=\frac{2}{\sqrt{\pi}} \int_{0}^{x} \exp \left(-s^{2}\right) d s
$$

and ${ }_{1} F_{1}(a ; b ; x)$ denotes the confluent hypergeometric function defined by

$$
{ }_{1} F_{1}(a ; b ; x)=\sum_{k=0}^{\infty} \frac{(a)_{k}}{(b)_{k}} \frac{x^{k}}{k !}
$$

The corresponding pdf can be expressed as

$$
f_{T}(t)=\frac{b^{a} d^{c} \Gamma\left(a+c-\frac{1}{2}\right)}{\sqrt{2 \pi} \Gamma(a+c)} t^{-\frac{1}{2}}\left(\frac{t}{2}+d\right)_{2}^{\frac{1}{2}-a-c}{ }_{2} F_{1}\left(a, a+c-\frac{1}{2} ; a+c ; \frac{d-b}{\frac{t}{2}+d}\right)
$$


and

$$
f_{T}(t)=\frac{b^{a} d^{c} \Gamma\left(a+c-\frac{1}{2}\right)}{\sqrt{2 \pi} \Gamma(a+c)} t^{-\frac{1}{2}}\left(\frac{t}{2}+b\right)_{2}^{\frac{1}{2}-a-c} F_{1}\left(c, a+c-\frac{1}{2} ; a+c ; \frac{b-d}{\frac{t}{2}+b}\right)
$$

for $\left|\frac{d-b}{\frac{t}{2}+d}\right|<1$ and $\left|\frac{b-d}{\frac{t}{2}+b}\right|<1$, respectively. The result given by (7) and (8) appears equivalent to (3) due to Net et al. (1990).

\subsection{The case $b=d$}

Suppose $b>0$ and $d>0$ are equal and take a real value. Then the cdf and pdf of $T$ can be expressed as

$$
F_{T}(t)=\frac{2 b^{a+c}}{\Gamma(a+c)} I(2 a+2 c-1, b, \sqrt{t / 2})
$$

and

$$
f_{T}(t)=\frac{b^{a+c} \Gamma\left(a+c-\frac{1}{2}\right)}{\sqrt{2 \pi} \Gamma(a+c)} t^{-\frac{1}{2}}\left(\frac{t}{2}+d\right)^{\frac{1}{2}-a-c},
$$

respectively, for $t>0$, where $I(n, p, c)$ is an elementary form defined by Lemma B1.

\subsection{The case $m$ odd}

Suppose $m>1$ is an odd integer. If in addition $n>1$ is also an odd integer then the cdf of $T$ can be expressed as

$$
\begin{gathered}
F_{T}(t)=\frac{2 b^{a} d^{c}(1-a-c)_{a}}{(a+c-1)(a-1) ! t^{a+c}}\left[\sum_{k=0}^{c-1} \frac{(1-c)_{k}(d-b)^{k}}{k !(2-a-c)_{k} t^{k}} I\left(2 a+2 c+2 k-1, \frac{d}{t}, \frac{1}{\sqrt{2}}\right)\right. \\
\left.-\sum_{k=0}^{a-1} \frac{(1-a)_{k}(b-d)^{k}}{k !(2-a-c)_{k} t^{k}} I\left(2 a+2 c+2 k-1, \frac{b}{t}, \frac{1}{\sqrt{2}}\right)\right]
\end{gathered}
$$

for $t>0$, where $I(n, p, c)$ is an elementary form defined by Lemma B1. On the other hand, if $n>1$ is an even integer then the cdf of $T$ can be expressed as

$$
\begin{aligned}
F_{T}(t)=\frac{2 c b^{a} d^{c}(1+c)_{a-1}}{(a-1) !} & \cdot\left[\sqrt{\pi}(d-b)^{-c} \sum_{p=0}^{a-1}(b-d)^{-p}\left(\begin{array}{c}
a-1 \\
p
\end{array}\right)\left(\frac{1}{2}\right)_{c+p-\frac{1}{2}} J\left(2 a-2 p-1, b, \sqrt{t}, \sqrt{\frac{d-b}{2}}\right)\right. \\
& \left.-\sum_{p=0}^{a-1} \frac{(-1)^{p}\left(\begin{array}{c}
a-1 \\
p
\end{array}\right)}{c+p} \sum_{k=1}^{c+p-\frac{1}{2}}(b-d)^{-k}(-c-p)_{k} I\left(2 a+2 c-2 k-1, d, \sqrt{\frac{t}{2}}\right)\right]
\end{aligned}
$$


and

$$
\begin{aligned}
F_{T}(t)= & \frac{2 c b^{a} d^{c}(1+c)_{a-1}}{(a-1) !}\left[\sqrt{\pi}(d-b)^{-c}\right. \\
& \cdot \sum_{p=0}^{a-1}(b-d)^{-p}\left(\begin{array}{c}
a-1 \\
p
\end{array}\right)\left(\frac{1}{2}\right)_{c+p-\frac{1}{2}} K\left(2 a-2 p-1, b, \sqrt{t}, \sqrt{\frac{b-d}{2}}\right) \\
& \left.\quad-\sum_{p=0}^{a-1} \frac{(-1)^{p}\left(\begin{array}{c}
a-1 \\
p
\end{array}\right)}{c+p} \sum_{k=1}^{c+p-\frac{1}{2}}(b-d)^{-k}(-c-p)_{k} I\left(2 a+2 c-2 k-1, d, \sqrt{\frac{t}{2}}\right)\right]
\end{aligned}
$$

for $d>b$ and $b>d$, respectively, and $t>0$, where $I(n, p, c), J(n, p, b, c)$ and $K(n, p, b, c)$ are elementary forms defined by Lemmas B1, B2 and B3. In either case $(n>1$ is an odd integer or an even integer), the corresponding pdf can be expressed as

$$
\begin{aligned}
f_{T}(t)=\frac{b^{a} d^{c} \Gamma\left(a+c-\frac{1}{2}\right)}{\sqrt{2 \pi} \Gamma(a+c)} t^{-\frac{1}{2}}\left(\frac{t}{2}+d\right)^{\frac{1}{2}-a-c}(1-y)^{-a} \\
\cdot\left[\frac{2+a+c}{2}(y-1) C_{a+1}\left(a, \frac{1}{2}, a+c ; \frac{y}{y-1}\right) \sum_{k=0}^{3} \frac{\left(\frac{1}{2}\right)_{k}(-3)_{k} y^{k}}{(a+c)_{k} k !(y-1)^{k}}\right. \\
\left.+C_{a+2}\left(a, \frac{1}{2}, a+c ; \frac{y}{y-1}\right) \sum_{k=0}^{2} \frac{\left(\frac{1}{2}\right)_{k}(-2)_{k} y^{k}}{(a+c)_{k} k !(y-1)^{k}}\right]
\end{aligned}
$$

and

$$
\begin{aligned}
f_{T}(t)=\frac{b^{a} d^{c} \Gamma\left(a+c-\frac{1}{2}\right)}{\sqrt{2 \pi} \Gamma(a+c)} t^{-\frac{1}{2}}\left(\frac{t}{2}+b\right)^{\frac{1}{2}-a-c}(1-y)^{\frac{1}{2}-c} \\
\cdot\left[\frac{2+a+c}{2(y-1)} C_{a+1}\left(a, \frac{1}{2}, a+c ; y\right) \sum_{k=0}^{3} \frac{\left(\frac{1}{2}\right)_{k}(-3)_{k} y^{k}}{(a+c)_{k} k !}\right. \\
\left.+C_{a+2}\left(a, \frac{1}{2}, a+c ; y\right) \sum_{k=0}^{2} \frac{\left(\frac{1}{2}\right)_{k}(-2)_{k} y^{k}}{(a+c)_{k} k !}\right]
\end{aligned}
$$

for $y=\frac{d-b}{\frac{t}{2}+d}<1$ and $y=\frac{b-d}{\frac{t}{2}+b}<1$, respectively, where $C_{i}(\alpha, \beta, \gamma, z)$ satisfies

$C_{i}(\alpha, \beta, \gamma, z)=-\frac{-2 \beta+\gamma+2 i+(\beta-\alpha-i) z}{(i-\beta)(\gamma-1)} C_{i-1}(\alpha, \beta, \gamma, z)+\frac{i-\beta+\gamma-1}{(i-\beta-1)(z-1)} C_{i-2}(\alpha, \beta, \gamma, z)$

with the initial values

$$
C_{0}(\alpha, \beta, \gamma, z)=1, \quad C_{1}(\alpha, \beta, \gamma, z)=\frac{2-2 \beta+\gamma+(\beta-\alpha-1) z}{(\beta-1)(z-1)} .
$$




\subsection{The case $n$ odd}

Suppose $n>1$ is an odd integer. If in addition $m>1$ is an even integer then the cdf of $T$ can be expressed as

$$
\begin{aligned}
F_{T}(t)= & \frac{2 a b^{a} d^{c}(1+a)_{c-1}}{(c-1) !} \\
& \cdot\left[\sqrt{\pi}(b-d)^{-a} \sum_{p=0}^{c-1}(d-b)^{-p}\left(\begin{array}{c}
c-1 \\
p
\end{array}\right)\left(\frac{1}{2}\right)_{a+p-\frac{1}{2}} J\left(2 c-2 p-1, d, \sqrt{t}, \sqrt{\frac{b-d}{2}}\right)\right. \\
& \left.-\sum_{p=0}^{c-1} \frac{(-1)^{p}\left(\begin{array}{c}
c-1 \\
p
\end{array}\right)}{a+p} \sum_{k=1}^{a+p-\frac{1}{2}}(d-b)^{-k}(-a-p)_{k} I\left(2 a+2 c-2 k-1, b, \sqrt{\frac{t}{2}}\right)\right]
\end{aligned}
$$

and

$$
\begin{aligned}
F_{T}(t)= & \frac{2 a b^{a} d^{c}(1+a)_{c-1}}{(c-1) !}\left[\sqrt{\pi}(b-d)^{-a}\right. \\
& \quad \sum_{p=0}^{c-1}(d-b)^{-p}\left(\begin{array}{c}
c-1 \\
p
\end{array}\right)\left(\frac{1}{2}\right)_{a+p-\frac{1}{2}} K\left(2 c-2 p-1, d, \sqrt{t}, \sqrt{\frac{d-b}{2}}\right) \\
& \left.\quad-\sum_{p=0}^{c-1} \frac{(-1)^{p}\left(\begin{array}{c}
c-1 \\
p
\end{array}\right)}{a+p} \sum_{k=1}^{a+p-\frac{1}{2}}(d-b)^{-k}(-a-p)_{k} I\left(2 a+2 c-2 k-1, b, \sqrt{\frac{t}{2}}\right)\right]
\end{aligned}
$$

for $b>d$ and $d>b$, respectively, and $t>0$, where $I(n, p, c), J(n, p, b, c)$ and $K(n, p, b, c)$ are elementary forms defined by Lemmas B1, B2 and B3. Whether or not $m>1$ is an even integer, the corresponding pdf can be expressed as

$$
\begin{aligned}
f_{T}(t)=\frac{b^{a} d^{c} \Gamma\left(a+c-\frac{1}{2}\right)}{\sqrt{2 \pi} \Gamma(a+c)} t^{-\frac{1}{2}}\left(\frac{t}{2}+d\right)^{\frac{1}{2}-a-c}(1-y)^{\frac{1}{2}-a} \\
\cdot\left[\frac{2+a+c}{2(y-1)} C_{c+1}\left(c, \frac{1}{2}, a+c ; y\right) \sum_{k=0}^{3} \frac{\left(\frac{1}{2}\right)_{k}(-3)_{k} y^{k}}{(a+c)_{k} k !}\right. \\
\left.+C_{c+2}\left(c, \frac{1}{2}, a+c ; y\right) \sum_{k=0}^{2} \frac{\left(\frac{1}{2}\right)_{k}(-2)_{k} y^{k}}{(a+c)_{k} k !}\right]
\end{aligned}
$$

and

$$
\begin{aligned}
f_{T}(t)=\frac{b^{a} d^{c} \Gamma\left(a+c-\frac{1}{2}\right)}{\sqrt{2 \pi} \Gamma(a+c)} t^{-\frac{1}{2}}\left(\frac{t}{2}+b\right)^{\frac{1}{2}-a-c}(1-y)^{-c} \\
\quad \cdot\left[\frac{2+a+c}{2}(y-1) C_{c+1}\left(c, \frac{1}{2}, a+c ; \frac{y}{y-1}\right) \sum_{k=0}^{3} \frac{\left(\frac{1}{2}\right)_{k}(-3)_{k} y^{k}}{(a+c)_{k} k !(y-1)^{k}}\right. \\
\left.+C_{c+2}\left(c, \frac{1}{2}, a+c ; \frac{y}{y-1}\right) \sum_{k=0}^{2} \frac{\left(\frac{1}{2}\right)_{k}(-2)_{k} y^{k}}{(a+c)_{k} k !(y-1)^{k}}\right]
\end{aligned}
$$


for $y=\frac{d-b}{\frac{t}{2}+d}<1$ and $y=\frac{b-d}{\frac{t}{2}+b}<1$, respectively, where $C_{i}(\alpha, \beta, \gamma, z)$ is as defined in (16)-(17).

\subsection{The case $m$ even, $n$ even}

Suppose both $m>1$ and $n>1$ are even integers. Then the cdf of $T$ can be expressed as

$$
\begin{aligned}
F_{T}(t)= & \frac{2^{1-a-c} b^{a} d^{c}\left(a-\frac{1}{2}\right) ! \Gamma(a)}{\left(\frac{1}{2}\right)_{a+c-1}\left(\frac{1}{2}\right)_{a-\frac{1}{2}}} \sum_{k=0}^{a-\frac{1}{2}} \sum_{p=0}^{k+a+c-1} \sum_{q=0}^{p} \\
& \cdot \sum_{\ell=0}^{a-\frac{1}{2}-k} \frac{(-1)^{p} 2^{-k-p}\left(\frac{1}{2}+k-a\right)_{\ell}(b-d)^{\ell}}{\left(a-\frac{1}{2}-k\right) ! \Gamma\left(k+\ell+\frac{1}{2}\right) k ! \ell !}\left(\begin{array}{c}
k+a+c-1 \\
p
\end{array}\right)\left(\begin{array}{l}
p \\
q
\end{array}\right) I_{1}(t)
\end{aligned}
$$

for $t>0$, where

$$
I_{1}(t)=\int_{0}^{\infty} z^{a+c+k+\ell-1} \exp \left(-\frac{b+d}{2} z\right) \operatorname{erf}(\sqrt{t z / 2}) I_{p-2 q}\left(\frac{(d-b) z}{2}\right) d z,
$$

where $I_{\nu}(\cdot)$ denotes the modified Bessel function of the first kind of order $\nu$ defined by

$$
I_{\nu}(z)=\sum_{k=0}^{\infty} \frac{1}{\Gamma(k+\nu+1) k !}\left(\frac{z}{2}\right)^{2 k+\nu} .
$$

If $m \geq 4$ is even and $n \geq 4$ is even then the corresponding pdf can be expressed as

$$
\begin{aligned}
f_{T}(t)=\frac{b^{a} d^{c} \Gamma\left(a+c-\frac{1}{2}\right)}{\sqrt{2 \pi} \Gamma(a+c)} t^{-\frac{1}{2}}\left(\frac{t}{2}+d\right)^{\frac{1}{2}-a-c}(1-y)^{-a} \\
\quad \cdot\left[\frac{4}{a-2} C_{a+c-3}^{\prime}\left(a, \frac{1}{2}, a+c, z\right) C_{a-\frac{1}{2}}\left(\frac{1}{2}, a, 1, z\right){ }_{2} F_{1}\left(\frac{1}{2},-\frac{3}{2} ; 1 ; z\right)\right. \\
\quad+\frac{4}{3(a-2)(y-1)} C_{a+c-3}^{\prime}\left(a, \frac{1}{2}, a+c, z\right) C_{a+\frac{1}{2}}\left(\frac{1}{2}, a, 1, z\right){ }_{2} F_{1}\left(\frac{1}{2},-\frac{1}{2} ; 1 ; z\right) \\
\quad+5(y-1) C_{a+c-2}^{\prime}\left(a, \frac{1}{2}, a+c, z\right) C_{a-\frac{1}{2}}\left(\frac{1}{2}, a, 2, z\right){ }_{2} F_{1}\left(\frac{1}{2},-\frac{3}{2} ; 2 ; z\right) \\
\left.\quad+C_{a+c-2}^{\prime}\left(a, \frac{1}{2}, a+c, z\right) C_{a+\frac{1}{2}}\left(\frac{1}{2}, a, 2, z\right){ }_{2} F_{1}\left(\frac{1}{2},-\frac{1}{2} ; 2 ; z\right)\right]
\end{aligned}
$$

and

$$
\begin{aligned}
f_{T}(t)=\frac{b^{a} d^{c} \Gamma\left(a+c-\frac{1}{2}\right)}{\sqrt{2 \pi} \Gamma(a+c)} t^{-\frac{1}{2}}\left(\frac{t}{2}+b\right)^{\frac{1}{2}-a-c}(1-y)^{\frac{1}{2}-c} \\
\quad \cdot\left[\frac{4}{a-2} C_{a+c-3}^{\prime}\left(a, \frac{1}{2}, a+c, y\right) C_{a-\frac{1}{2}}\left(\frac{1}{2}, a, 1, y\right){ }_{2} F_{1}\left(\frac{1}{2},-\frac{3}{2} ; 1 ; y\right)\right. \\
\quad+\frac{4(y-1)}{3(a-2)} C_{a+c-3}^{\prime}\left(a, \frac{1}{2}, a+c, y\right) C_{a+\frac{1}{2}}\left(\frac{1}{2}, a, 1, y\right){ }_{2} F_{1}\left(\frac{1}{2},-\frac{1}{2} ; 1 ; y\right) \\
\quad+\frac{5}{y-1} C_{a+c-2}^{\prime}\left(a, \frac{1}{2}, a+c, y\right) C_{a-\frac{1}{2}}\left(\frac{1}{2}, a, 2, y\right){ }_{2} F_{1}\left(\frac{1}{2},-\frac{3}{2} ; 2 ; y\right) \\
\left.\quad+C_{a+c-2}^{\prime}\left(a, \frac{1}{2}, a+c, y\right) C_{a+\frac{1}{2}}\left(\frac{1}{2}, a, 2, y\right){ }_{2} F_{1}\left(\frac{1}{2},-\frac{1}{2} ; 2 ; y\right)\right]
\end{aligned}
$$


for $y=\frac{d-b}{\frac{t}{2}+d}<1$ and $y=\frac{b-d}{\frac{t}{2}+b}<1$, respectively, where $z=\frac{y}{y-1}, C_{i}(\alpha, \beta, \gamma, z)$ is as defined in (16)-(17) and $C_{i}^{\prime}(\alpha, \beta, \gamma, z)$ satisfies

$$
\begin{aligned}
C_{i}^{\prime}(\alpha, \beta, \gamma, z)= & \frac{(i-\gamma)(i-\gamma-1)(1-z)}{(\alpha-\gamma+i-1)(\beta-\gamma+i-1) z} C_{i-2}^{\prime}(\alpha, \beta, \gamma, z) \\
& \quad+\frac{(\gamma-i)\{1-\gamma+i-[\alpha+\beta+2(i-\gamma-1)+3] z\}}{(\alpha-\gamma+i)(\beta-\gamma+i) z} C_{i-1}^{\prime}(\alpha, \beta, \gamma, z)
\end{aligned}
$$

with the initial values

$$
C_{0}^{\prime}(\alpha, \beta, \gamma, z)=1, \quad C_{1}^{\prime}(\alpha, \beta, \gamma, z)=\frac{(\gamma-1)[2-\gamma-(\alpha+\beta-2 \gamma+3) z]}{(\alpha-\gamma+1)(\beta-\gamma+1) z} .
$$

By Prudnikov et al. (1986, volume 3, Section 7.3.2),

$$
\begin{aligned}
& { }_{2} F_{1}\left(\frac{1}{2}, \frac{1}{2} ; 1 ; z\right)=\frac{2}{\pi} \mathbf{K}(z), \\
& { }_{2} F_{1}\left(\frac{3}{2}, \frac{1}{2} ; 3 ; z\right)=\frac{4}{\pi z}[\mathbf{K}(z)-\mathbf{E}(z)], \\
& { }_{2} F_{1}\left(\frac{1}{2}, \frac{1}{2} ; 2 ; z\right)=\frac{4}{\pi z}[\mathbf{E}(z)+(z-1) \mathbf{K}(z)], \\
& { }_{2} F_{1}\left(\frac{1}{2},-\frac{3}{2} ; 1 ; z\right)=\frac{1}{3 \pi}[2(z-1) \mathbf{K}(z)-4(z-2) \mathbf{E}(z)], \\
& { }_{2} F_{1}\left(\frac{1}{2},-\frac{1}{2} ; 1 ; z\right)=\frac{2}{\pi} \mathbf{E}(z), \\
& { }_{2} F_{1}\left(\frac{1}{2},-\frac{3}{2} ; 2 ; z\right)=\frac{4}{15 \pi z}\{[(7-2 z) z+3] \mathbf{E}(z)+(z-1)(z+3) \mathbf{K}(z)\}, \\
& { }_{2} F_{1}\left(\frac{1}{2},-\frac{1}{2} ; 2 ; z\right)=\frac{4}{3 \pi z}[(z+1) \mathbf{E}(z)+(z-1) \mathbf{K}(z)],
\end{aligned}
$$

where $\mathbf{K}(\cdot)$ and $\mathbf{E}(\cdot)$ are complete elliptical integrals of the first and second kinds defined by

$$
\mathbf{K}(z)=\int_{0}^{\frac{\pi}{2}}\left(1-z \sin ^{2} t\right)^{-\frac{1}{2}} d t
$$

and

$$
\mathbf{E}(z)=\int_{0}^{\frac{\pi}{2}}\left(1-z \sin ^{2} t\right)^{\frac{1}{2}} d t
$$

respectively.

Hence, the pdf of $T$ for any $m \geq 2$ even and $n \geq 2$ even can be expressed in terms of $\mathbf{K}(z)$ and $\mathbf{E}(z)$.

\section{Discussion}

Here, we illustrate the practical use and efficiency of the expressions in Sections 2.2 to 2.5. We do this by comparing: the pdf of $T$ suggested by Sections 2.2 to 2.5 with the pdf of $T$ suggested by the Student's $t$ approximation with degree of freedom (2); the cdf of $T$ suggested by Sections 2.2 to 2.5 with the cdf of $T$ suggested by the Student's $t$ approximation with degree of freedom (2); the power function of $\mu_{X}-\mu_{Y}$ suggested by Sections 2.2 to 2.5 with the power function of $\mu_{X}-\mu_{Y}$ suggested by the Student's $t$ approximation with degree of freedom (2); the central processing unit times taken to compute the pdfs of $T$ in Sections 2.3-2.4 with the central processing unit 
times taken to compute the corresponding pdfs in Section 2.1; the central processing unit times taken to compute the cdfs of $T$ in Sections 2.3-2.4 with the central processing unit times taken to compute the corresponding cdfs in Section 2.1.

Suppose $\mu_{X}=\mu_{Y}$. Then the pdf and the cdf of $T$ suggested by the Student's $t$ approximation with degree of freedom (2) are

$$
f_{T, \operatorname{Approx}}(t)=t^{-\frac{1}{2}} f_{t_{\nu}}(\sqrt{t})
$$

and

$$
F_{T, \text { Approx }}(t)=F_{t_{\nu}}(\sqrt{t})-F_{t_{\nu}}(-\sqrt{t}),
$$

respectively, where $f_{t_{\nu}}(\cdot)$ and $F_{t_{\nu}}(\cdot)$ denote, respectively, the pdf and the cdf of a Student's $t$ random variable with degree of freedom $\nu$. The exact expressions for the pdf and the cdf of $T$ given in Section 2 involve the unknown parameters $\sigma_{X}^{2}$ and $\sigma_{Y}^{2}$. We also computed them with $\sigma_{X}^{2}$ and $\sigma_{Y}^{2}$ replaced by the sample variances $s_{X}^{2}$ and $s_{Y}^{2}$, respectively, yielding what we refer to as the "estimated exact" pdfs and cdfs of $T$. The $s_{X}^{2}$ was computed as the variance of a single sample of size $m$ simulated from a normal distribution with variance $\sigma_{X}^{2}$ and $s_{Y}^{2}$ was computed as the variance of a single sample of size $n$ simulated from a normal distribution with variance $\sigma_{Y}^{2}$. Note that the estimated exact pdf and the estimated exact cdf are still continuous functions of $t$ just like $f_{T}(t)$ and $F_{T}(t)$ are.

The exact, estimated exact and approximate pdfs of $T$ are plotted in Figure 1 for a range of different values of $m$ and $n$. The exact, estimated exact and approximate cdfs of $T$ are plotted in Figure 2 for a range of different values of $m$ and $n$.

Now consider testing $H_{0}: \mu_{X}=\mu_{Y}$ versus $H_{1}: \mu_{X} \neq \mu_{Y}$ with significance level $\alpha=0.05$. The rule for rejecting $H_{0}$ suggested by the Student's $t$ approximation with degree of freedom (2) is

$$
\frac{|\bar{x}-\bar{y}|}{\sqrt{\frac{s_{X}^{2}}{m}+\frac{s_{Y}^{2}}{n}}}>F_{t_{\nu}}^{-1}\left(1-\frac{\alpha}{2}\right) .
$$

The rule for rejecting $H_{0}$ suggested by the results in Section 2 is

$$
\frac{(\bar{x}-\bar{y})^{2}}{\frac{s_{X}^{2}}{m}+\frac{s_{Y}^{2}}{n}}>F_{T}^{-1}(1-\alpha) .
$$

$F_{T}^{-1}\left(\frac{\alpha}{2}\right)$ and $F_{T}^{-1}\left(1-\frac{\alpha}{2}\right)$ involve the unknown parameters, $\sigma_{X}^{2}$ and $\sigma_{Y}^{2}$. With them replaced by the sample variances, we obtain the rule

$$
\frac{(\bar{x}-\bar{y})^{2}}{\frac{s_{X}^{2}}{m}+\frac{s_{Y}^{2}}{n}}>\widehat{F}_{T}^{-1}(1-\alpha),
$$

where $\widehat{F}_{T}^{-1}\left(\frac{\alpha}{2}\right)$ and $\widehat{F}_{T}^{-1}\left(1-\frac{\alpha}{2}\right)$ denote the estimates of $F_{T}^{-1}\left(\frac{\alpha}{2}\right)$ and $F_{T}^{-1}\left(1-\frac{\alpha}{2}\right)$, respectively. Finally, let $\widetilde{F}(\cdot)$ denote the empirical cdf of $|\bar{x}-\bar{y}| / \sqrt{\frac{s_{X}^{2}}{m}+\frac{s_{Y}^{2}}{n}}$ computed over ten thousand samples simulated from $N\left(\mu_{X}, \sigma_{X}^{2}\right)$ and $N\left(\mu_{Y}, \sigma_{Y}^{2}\right)$. Then a rule for rejecting $H_{0}$ is

$$
\frac{|\bar{x}-\bar{y}|}{\sqrt{\frac{s_{X}^{2}}{m}+\frac{s_{Y}^{2}}{n}}}>\widetilde{F}^{-1}(1-\alpha) .
$$

The power functions corresponding to (25)-(28) are plotted in Figure 3 for a range of different values of $m$ and $n$. "Approx" power refers to (25), "Exact" power refers to (26), "Estimated 
Figure 1: Exact, estimated exact and approximate pdfs of $T$ in $(5)$ versus $t=0.01,0.02, \ldots, 10$ when $\sigma_{X}^{2}=4$ and $\sigma_{Y}^{2}=1: m=5$ and $n=3$ (top left), $m=3$ and $n=4$ (top right), $m=4$ and $n=3$ (middle left), $m=2$ and $n=4$ (middle right), $m=n=3$ (bottom left), $m=n=2$ (bottom right). Both axes are plotted in log scale.

Figure 2: Exact, estimated exact and approximate cdfs of $T$ in (5) versus $t=0.01,0.02, \ldots, 10$ when $\sigma_{X}^{2}=4$ and $\sigma_{Y}^{2}=1: m=5$ and $n=3$ (top left), $m=3$ and $n=4$ (top right), $m=4$ and $n=3$ (middle left), $m=2$ and $n=4$ (middle right), $m=n=3$ (bottom left), $m=n=2$ (bottom right).

Exact" power refers to (27) and "Simulated" power refers to (28). The cdf of $T$ needed for (26) and (27) were computed using: (9) when $m=n$; (11) when $m$ and $n \neq m$ are odd; (12)-(13) when $m$ is odd and $n \neq m$ is even; (18)-(19) when $m$ is even and $n \neq m$ is odd; (22) when $m$ and $n \neq m$ are even.

Figure 4 plots the central processing unit times taken to compute the elementary expressions in Sections 2.3-2.4 and the expressions due to Nel et al. (1990). These times are plotted versus a range of $m$ and $n$. "Nel et al.'s PDF" in Figure 4 refers to (3). "Nel et al.'s CDF" refers to the integrated version of (3), integration performed numerically. "Proposed PDF" refers to one of (14) or (15) if $m$ is odd. "Proposed PDF" refers to one of (20) or (21) if $n$ is odd. "Proposed CDF" refers to one of (11), (12) or (13) if $m$ is odd. "Proposed CDF" refers to one of (18) or (19) if $n$ is odd.

In Figure 1, the exact and estimated exact pdfs for $m=n$ were computed using (10). Those when $m$ and $n \neq m$ are odd were computed using (14)-(15). Those when $m$ is odd and $n \neq m$ is even were computed using (14)-(15). Those when $m$ is even and $n \neq m$ is odd were computed using (20)-(21). Those when $m$ and $n \neq m$ are even were computed using (23)-(24). In Figure 2 , the exact and estimated exact cdfs for $m=n$ were computed using (9). Those when $m$ and $n \neq m$ are odd were computed using (11). Those when $m$ is odd and $n \neq m$ is even were computed using (12)-(13). Those when $m$ is even and $n \neq m$ is odd were computed using (18)-(19). Those when $m$ and $n \neq m$ are even were computed using (22). The approximate pdfs and cdfs in Figures 1 and 2 are based on the Student's $t$ approximation. All computations were performed in Maple with Digits set equal to 20. Maple like most other algebraic manipulation packages allows for arbitrary precision, so the accuracy of computations was not an issue. The inversions needed for (26) and (27) were performed using fsolve.

Figure 1 shows that the approximate pdf of $T$ differs significantly from the exact one for all sufficiently large values of $t$. The estimated exact pdf of $T$ does not differ as much from the exact one as the approximate pdf of $T$ does. The estimated exact pdf appears a lot closer to the exact pdf especially in the upper tail of each of the six plots in Figure 1. This could be significant when the extremes of the means of two random samples are of interest, for example, the extremes of mean returns of two different stocks, the extremes of mean rainfall for two different locations, the extremes of mean wind speeds for two different locations, the extremes of mean sea levels at two different coastal points, the extremes of mean exchange rates for two different countries, and so on.

Figure 2 shows that the approximate cdf of $T$ is the furthest from the exact one for all values of $t$. The estimated exact cdf of $T$ does not differ as much from the exact one as the approximate cdf of $T$ does. The estimated exact cdf appears a lot closer to the exact cdf in each of the six

Figure 3: Exact, estimated exact, approximate and simulated power of $\mu_{X}-\mu_{Y}$ when $\sigma_{X}^{2}=4$ and $\sigma_{Y}^{2}=1: m=5$ and $n=3$ (top left), $m=3$ and $n=4$ (top right), $m=4$ and $n=3$ (middle left), $m=2$ and $n=4$ (middle right), $m=n=3$ (bottom left), $m=n=2$ (bottom right). 
Figure 4: Central processing unit times to compute pdf of $T$ one hundred times versus $n=$ $3,4, \ldots, 101$ when $m=3, \sigma_{X}^{2}=4$ and $\sigma_{Y}^{2}=1$ (top left); Central processing unit times to compute pdf of $T$ one hundred times versus $m=3,4, \ldots, 101$ when $n=3, \sigma_{X}^{2}=4$ and $\sigma_{Y}^{2}=1$ (top right); Central processing unit times to compute cdf of $T$ one hundred times versus $n=3,4, \ldots, 101$ when $m=3, \sigma_{X}^{2}=4$ and $\sigma_{Y}^{2}=1$ (bottom left); Central processing unit times to compute cdf of $T$ one hundred times versus $m=3,4, \ldots, 101$ when $n=3, \sigma_{X}^{2}=4$ and $\sigma_{Y}^{2}=1$ (bottom right).

plots in Figure 2. Figure 3 shows that (26) and (27) give the best power for most values of $\mu_{X}-\mu_{Y} \neq 0$. (25) gives the worst power for most values of $\mu_{X}-\mu_{Y} \neq 0$. The powers given by (26) and (27) do not differ much from the power given by the simulated version (28). Figure 4 shows that the elementary expressions in Sections 2.3-2.4 are computationally more efficient than the expressions in Section 2.1 for every $m$ and every $n$. As expected, the central processing unit times increase with increasing $m$. They also increase with increasing $n$.

The observations in Figures 1 to 3 were noted also for a wide range of other small values of $m$ and $n$ (equal and unequal sample sizes) and for a wide range of other values of $\sigma_{X}^{2}$ and $\sigma_{Y}^{2}$ (equal and unequal variances). Hence, the use of the Student's $t$ approximation in these cases can lead to serious errors and less power.

The observations in Figure 4 were noted also for a wide range of other values of $m$ and $n$ (equal and unequal sample sizes) and for a wide range of other values of $\sigma_{X}^{2}$ and $\sigma_{Y}^{2}$ (equal and unequal variances). Hence, the use of Nel et al.'s expression is computationally less efficient and computationally less convenient than the elementary expressions in Sections 2.3-2.4.

Some future work are: i) derive elementary forms of the exact distribution of (4) corresponding to non-integer values of $m$ and $n$; ii) consider one-sided versions of (4) and derive their exact distributions, (4) corresponds to a two-sided test of the equality $\mu_{X}=\mu_{Y}$; iii) develop distribution theory for the effect of replacing $\sigma_{X}^{2}$ and $\sigma_{Y}^{2}$ by their sample counterparts.

\section{Acknowledgments}

The authors would like to thank the Editor and the two referees for careful reading and comments which greatly improved the paper.

\section{References}

[1] Fisher, R. A. (1935). The fiducial argument in statistical inference. Annals of Eugenics, 6, 391-398.

[2] Gradshteyn, I. S. and Ryzhik, I. M. (2000). Table of Integrals, Series, and Products, sixth edition. Academic Press, San Diego.

[3] Johnson, N. L., Kotz, S. and Balakrishnan, N. (1995). Continuous Univariate Distributions, volume 2, second edition. John Wiley and Sons, New York.

[4] Kakizawa, Y. and Iwashita, T. (2008). Hotelling's one-sample and two-sample $T^{2}$ tests and the multivariate Behrens-Fisher problem under nonnormality. Journal of Statistical Planning and Inference, 138, 3379-3404.

[5] Kim, S. -H. and Cohen, A. S. (1998). On the Behrens-Fisher problem: A review. Journal of Educational and Behavioral Statistics, 23, 356-377.

[6] Krishnamoorthy, K. and Yu, J. (2004). Modified Nel and Van der Merwe test for the multivariate Behrens-Fisher problem. Statistics and Probability Letters, 66, 161-169.

[7] Nel, D. D., van der Merwe, C. A. and Moser, B. K. (1990). The exact distributions of the univariate and multivariate Behrens-Fisher statistics with a comparison of several solutions in the univariate case. Communications in Statistics - Theory and Methods, 19, $279-298$. 
[8] Prudnikov, A. P., Brychkov, Y. A. and Marichev, O. I. (1986). Integrals and Series, volumes 1, 2 and 3. Gordon and Breach Science Publishers, Amsterdam.

[9] R Development Core Team (2015). A Language and Environment for Statistical Computing: R Foundation for Statistical Computing. Vienna, Austria.

[10] Welch, B. L. (1938). The significance of the difference between two means when the population variances are unequal. Biometrika, 29, 350-362.

\section{Appendix A: The proofs}

The derivation of the results in Section 2 requires the following lemma which derives the exact pdf of the sum of $V \sim \Gamma(a, b)$ and $W \sim \Gamma(c, d)$.

Lemma A1 Suppose $V \sim \Gamma(a, b)$ and $W \sim \Gamma(c, d)$ are independent random variables. Then the pdf of $S=V+W$ can be expressed as

$$
f_{S}(s)=\frac{b^{a} d^{c}}{\Gamma(a+c)} s^{a+c-1} \exp (-d s)_{1} F_{1}(a ; a+c ;-(b-d) s)
$$

for $s>0$.

Proof: We can write

$$
\begin{aligned}
f_{S}(s) & =\int_{0}^{s} f_{V}(v) f_{W}(s-v) d v \\
& =\frac{b^{a} d^{c} \exp (-d s)}{\Gamma(a) \Gamma(c)} \int_{0}^{s} v^{a-1}(s-v)^{c-1} \exp [-(b-d) v] d v \\
& =\frac{b^{a} d^{c} s^{a+c-1} \exp (-d s)}{\Gamma(a) \Gamma(c)} \int_{0}^{1} v^{a-1}(1-v)^{c-1} \exp [-(b-d) s v] d v .
\end{aligned}
$$

The result follows by using equation (2.3.6.1) in Prudnikov et al. (1986, volume 1) to calculate the integral in (29).

Proof of (6): Using Lemma A1, we can write

$F_{T}(t)=\int_{0}^{\infty} F_{U}(t z) f_{S}(z) d z=\frac{b^{a} d^{c}}{\Gamma(a+c)} \int_{0}^{\infty} F_{U}(t z) z^{a+c-1} \exp (-d z)_{1} F_{1}(a ; a+c ;-(b-d) z) d z$.

The result follows since $F_{U}(u)=\operatorname{erf}(\sqrt{u / 2})$.

Proof of (7)-(8): Differentiating (6) with respect to $t$, we obtain

$$
f_{T}(t)=\frac{b^{a} d^{c}}{\sqrt{2 \pi} \Gamma(a+c)} t^{-\frac{1}{2}} \int_{0}^{\infty} z^{a+c-\frac{3}{2}} \exp \left[-\left(\frac{t}{2}+d\right) z\right]{ }_{1} F_{1}(a ; a+c ;-(b-d) z) d z .
$$

The results follow by using equation (7.621.4) in Gradshteyn and Ryzhik (2000) to calculate the integral in (30).

Proof of (9) and (10): If $b=d$ then (6) reduces to

$$
F_{T}(t)=\frac{b^{a} d^{c}}{\Gamma(a+c)} \int_{0}^{\infty} z^{a+c-1} \exp (-d z) \operatorname{erf}(\sqrt{t z / 2}) d z
$$

(9) follows by applying Lemma B1 to calculate the integral in (31). (10) follows from (7)-(8).

Proof of (11): Using the fact

$$
{ }_{1} F_{1}(i ; j ; z)=\frac{(i-2) !(1-i)_{j} z^{1-i}}{(i-1) !}\left[\sum_{k=0}^{j-i-1} \frac{(i-j+1)_{k} z^{k}}{k !(2-j)_{k}}-\exp (z) \sum_{k=0}^{i-1} \frac{(1-i)_{k}(-z)^{k}}{k !(2-j)_{k}}\right],
$$


see http://functions.wolfram.com/07.20.03.0024.01, rewrite the integrand of (6) as

$$
F_{T}(t)=\frac{b^{a} d^{c}(1-a-c)_{a}}{(a+c-1)(a-1) !}\left[\sum_{k=0}^{c-1} \frac{(1-c)_{k}(d-b)^{k}}{k !(2-a-c)_{k}} I_{1}-\sum_{k=0}^{a-1} \frac{(1-a)_{k}(b-d)^{k}}{k !(2-a-c)_{k}} I_{2}\right],
$$

where

$$
I_{1}=\int_{0}^{\infty} z^{a+c+k-1} \exp (-d z) \operatorname{erf}(\sqrt{t z / 2}) d z
$$

and

$$
I_{2}=\int_{0}^{\infty} z^{a+c+k-1} \exp (-b z) \operatorname{erf}(\sqrt{t z / 2}) d z .
$$

The result follows by applying Lemma B1 to calculate $I_{1}$ and $I_{2}$.

Proof of (12)-(13): Using the fact

$$
\begin{aligned}
{ }_{1} F_{1}\left(i ; j+\frac{1}{2} ; z\right)= & \frac{(2 j-2 i+1)}{2(i-1) !}\left(j-i+\frac{3}{2}\right)_{i-1} \\
& \cdot\left[\sqrt{\pi} \exp (z) z^{i-j-\frac{1}{2}} \operatorname{erf}(\sqrt{z}) \sum_{p=0}^{i-1}(-z)^{-p}\left(\begin{array}{c}
i-1 \\
p
\end{array}\right)\left(\frac{1}{2}\right)_{j-i+p}\right. \\
& \left.\quad-2 \sum_{p=0}^{i-1} \frac{(-1)^{p}\left(\begin{array}{c}
i-1 \\
p
\end{array}\right)}{2 j-2 i+2 p+1} \sum_{k=1}^{j-i+p}(-z)^{-k}\left(-j+i-p-\frac{1}{2}\right)_{k}\right],
\end{aligned}
$$

see http://functions.wolfram.com/07.20.03.0130.01, rewrite the integrand of (6) as

$$
\begin{aligned}
F_{T}(t)= & \frac{c b^{a} d^{c}(1+c)_{a-1}}{(a-1) !} \\
& \cdot\left[\sqrt{\pi}(d-b)^{-c} \sum_{p=0}^{a-1}(b-d)^{-p}\left(\begin{array}{c}
a-1 \\
p
\end{array}\right)\left(\frac{1}{2}\right)_{c+p-\frac{1}{2}} I_{1}\right. \\
& \left.\quad-\sum_{p=0}^{a-1} \frac{(-1)^{p}\left(\begin{array}{c}
a-1 \\
p
\end{array}\right)}{c+p} \sum_{k=1}^{c+p-\frac{1}{2}}(b-d)^{-k}(-c-p)_{k} I_{2}\right],
\end{aligned}
$$

where

$$
I_{1}=\int_{0}^{\infty} z^{a-1-p} \exp (-b z) \operatorname{erf}(\sqrt{t z}) \operatorname{erf}\left(\sqrt{\frac{(d-b) z}{2}}\right) d z
$$

and

$$
I_{2}=\int_{0}^{\infty} z^{a+c-k-1} \exp (-d z) \operatorname{erf}\left(\sqrt{\frac{t z}{2}}\right) d z
$$

$I_{1}$ can be calculated by Lemma B2 if $d>b . I_{1}$ can be calculated by Lemma B3 if $d<b . I_{2}$ can be calculated by Lemma B1. The result follows.

Proof of (18)-(19): Using the transformation ${ }_{1} F_{1}(\beta-\alpha ; \beta ; z)=\exp (z){ }_{1} F_{1}(\alpha ; \beta ;-z)$, see equation (7.2.2.8) in Prudnikov et al. (1986, volume 3), we can rewrite (6) as

$$
F_{T}(t)=\frac{b^{a} d^{c}}{\Gamma(a+c)} \int_{0}^{\infty} z^{a+c-1} \exp (-b z) \operatorname{erf}(\sqrt{t z / 2}){ }_{1} F_{1}(c ; a+c ;-(d-b) z) d z .
$$


Since $c$ is an integer and $a+c$ is a half integer, the arguments of the proof of (12)-(13) apply.

Proof of (22): Follows by using the fact

$$
\begin{aligned}
{ }_{1} F_{1}\left(i+\frac{1}{2} ; j ; z\right)= & \frac{2^{1-j} \exp (z / 2)(j-1) ! i !}{\left(\frac{1}{2}\right)_{j-1}\left(\frac{1}{2}\right)_{i}} \sum_{k=0} \frac{2^{-k}(-z)^{k}}{k !} L_{i-k}^{k-\frac{1}{2}}(-z) \\
& \cdot \sum_{p=0}^{k+j-1}(-1)^{p} 2^{-p}\left(\begin{array}{c}
k+j-1 \\
p
\end{array}\right) \sum_{q=0}^{p} I_{p-2 q}\left(\frac{z}{2}\right)
\end{aligned}
$$

where

$$
L_{n}^{\lambda}(z)=\frac{\Gamma(\lambda+n+1)}{n !} \sum_{k=0}^{n} \frac{(-n)_{k} z^{k}}{\Gamma(\lambda+k+1) k !},
$$

see http://functions.wolfram.com/07.20.03.0120.01 .

Proof of (14)-(15): By using the transformation formulas, ${ }_{2} F_{1}(\gamma-\alpha, \gamma-\beta ; \gamma ; z)=(1-$ $z)^{\alpha+\beta-\gamma}{ }_{2} F_{1}(\alpha, \beta ; \gamma ; z)$ and ${ }_{2} F_{1}\left(\alpha, \gamma-\beta ; \gamma ; \frac{z}{z-1}\right)=(1-z)_{2}{ }_{2} F_{1}(\alpha, \beta ; \gamma ; z)$, see equations (7.3.1.3), (7.3.1.4) in Prudnikov et al. (1986, volume 3), we can write

$$
{ }_{2} F_{1}\left(a, a+c-\frac{1}{2} ; a+c ; y\right)=(1-y)^{-a}{ }_{2} F_{1}\left(a, \frac{1}{2} ; a+c ; \frac{y}{y-1}\right)
$$

and

$$
{ }_{2} F_{1}\left(c, a+c-\frac{1}{2} ; a+c ; y\right)=(1-y)^{\frac{1}{2}-c}{ }_{2} F_{1}\left(a, \frac{1}{2} ; a+c ; y\right) .
$$

The results follow by using the following fact

$$
\begin{gathered}
{ }_{2} F_{1}(\alpha, \beta ; \gamma ; z)=\frac{i-\beta+\gamma}{(i-\beta)(z-1)} C_{i-1}(\alpha, \beta, \gamma, z){ }_{2} F_{1}(\alpha, \beta-i-1 ; \gamma ; z) \\
\quad+C_{i}(\alpha, \beta, \gamma, z){ }_{2} F_{1}(\alpha, \beta-i ; \gamma ; z)
\end{gathered}
$$

see Prudnikov et al. (1986, volume 3, Section 7.3.1), with $i=\alpha+2$.

Proof of (20)-(21): Similar to the proof of (14)-(15).

Proof of (23)-(24): By using the following fact

$$
\begin{gathered}
{ }_{2} F_{1}(\alpha, \beta ; \gamma ; z)=\frac{(i-\gamma+1)(\alpha-\gamma)(1-z)}{(i+\alpha-\gamma)(i+\beta-\gamma) z} C_{i-1}^{\prime}(\alpha, \beta, \gamma, z){ }_{2} F_{1}(\alpha, \beta ; \gamma-i-1 ; z) \\
\quad+C_{i}^{\prime}(\alpha, \beta, \gamma, z){ }_{2} F_{1}(\alpha, \beta ; \gamma-i ; z)
\end{gathered}
$$

see Prudnikov et al. (1986, volume 3, Section 7.3.1), with $i=\gamma-2$, we can express

$$
\begin{array}{r}
{ }_{2} F_{1}\left(a, \frac{1}{2} ; a+c ; z\right)=-\frac{4(1-z)}{3(a-2)} C_{a+c-3}^{\prime}\left(a, \frac{1}{2} ; a+c ; z\right){ }_{2} F_{1}\left(\frac{1}{2}, a ; 1 ; z\right) \\
+C_{a+c-2}^{\prime}\left(a, \frac{1}{2} ; a+c ; z\right){ }_{2} F_{1}\left(\frac{1}{2}, a ; 2 ; z\right) .
\end{array}
$$

Now applying (34) to each of the two Gauss hypergeometric terms on the right hand side of 
(35), we obtain

$$
\begin{aligned}
{ }_{2} F_{1}\left(a, \frac{1}{2} ; a+c ; z\right)= & \frac{4}{a-2} C_{a+c-3}^{\prime}\left(a, \frac{1}{2}, a+c, z\right) C_{a-\frac{1}{2}}\left(\frac{1}{2}, a, 1, z\right){ }_{2} F_{1}\left(\frac{1}{2},-\frac{3}{2} ; 1 ; z\right) \\
& +\frac{4(y-1)}{3(a-2)} C_{a+c-3}^{\prime}\left(a, \frac{1}{2}, a+c, z\right) C_{a+\frac{1}{2}}\left(\frac{1}{2}, a, 1, z\right){ }_{2} F_{1}\left(\frac{1}{2},-\frac{1}{2} ; 1 ; z\right) \\
& +\frac{5}{z-1} C_{a+c-2}^{\prime}\left(a, \frac{1}{2}, a+c, z\right) C_{a-\frac{1}{2}}\left(\frac{1}{2}, a, 2, z\right){ }_{2} F_{1}\left(\frac{1}{2},-\frac{3}{2} ; 2 ; z\right) \\
& +C_{a+c-2}^{\prime}\left(a, \frac{1}{2}, a+c, z\right) C_{a+\frac{1}{2}}\left(\frac{1}{2}, a, 2, z\right){ }_{2} F_{1}\left(\frac{1}{2},-\frac{1}{2} ; 2 ; z\right) .
\end{aligned}
$$

The results follow from (7), (8), (32) and (33).

\section{Appendix B: New integral identities}

Here, we give three integral identities. One of them (Lemma B1) is known. The other two (Lemmas B2 and B3) are new. Each lemma gives an elementary expression for the associated integral.

\section{Lemma B1 Let}

$$
I(n, p, b)=\int_{0}^{\infty} z^{n} \exp \left(-p z^{2}\right) \operatorname{erf}(b z) d z
$$

for $n \geq 1, \operatorname{Re}(p)>0$ and $\operatorname{Re}\left(p+b^{2}\right)>0$. Then

$$
I(2 m, p, b)=\frac{(-1)^{m}}{\sqrt{\pi}} \frac{\partial^{m}}{\partial p^{m}}\left[\frac{1}{\sqrt{p}} \arctan \left(\frac{b}{\sqrt{p}}\right)\right]
$$

and

$$
I(2 m+1, p, b)=\frac{(-1)^{m} c}{2} \frac{\partial^{m}}{\partial p^{m}}\left[\frac{1}{p \sqrt{p+b^{2}}}\right]
$$

for $m=0,1, \ldots$ with the initial values

$$
I(0, p, b)=\frac{1}{\sqrt{p \pi}} \arctan \left(\frac{b}{\sqrt{p}}\right)
$$

and

$$
I(1, p, b)=\frac{c}{2 p \sqrt{p+b^{2}}} .
$$

Proof: See equation (2.8.5.9) in Prudnikov et al. (1986, volume 2).

Lemma B2 Let

$$
J(n, p, b, c)=\int_{0}^{\infty} z^{n} \exp \left(-p z^{2}\right) \operatorname{erf}(b z) \operatorname{erf}(c z) d z
$$

for $n \geq 0, \operatorname{Re}(p)>0, \operatorname{Re}(b)>0$ and $\operatorname{Re}(c)>0$. Then

$$
J(n, p, b, c)=\frac{2 p}{n+1} J(n+2, p, b, c)-\frac{2\left[I\left(n+1, p+b^{2}, b\right)+I\left(n+1, p+b^{2}, c\right)\right]}{\sqrt{\pi}(n+1)}
$$

with the initial values

$$
J(0, p, b, c)=\frac{1}{\sqrt{\pi p}} \arctan \left(\frac{b c}{\sqrt{p} \sqrt{b^{2}+c^{2}+p}}\right)
$$


and

$$
J(1, p, b, c)=\frac{1}{\pi p}\left[\frac{b}{\sqrt{b^{2}+p}} \arctan \left(\frac{c}{\sqrt{b^{2}+p}}\right)+\frac{c}{\sqrt{c^{2}+p}} \arctan \left(\frac{b}{\sqrt{c^{2}+p}}\right)\right] .
$$

Proof: By integration by parts,

$$
\begin{aligned}
& \int_{0}^{\infty} z^{n} \exp \left(-p z^{2}\right) \operatorname{erf}(b z) \operatorname{erf}(c z) d z \\
= & {\left[\frac{z^{n+1}}{n+1} \exp \left(-p z^{2}\right) \operatorname{erf}(b z) \operatorname{erf}(c z)\right]_{0}^{\infty} } \\
& +\frac{2 p}{n+1} \int_{0}^{\infty} z^{n+2} \exp \left(-p z^{2}\right) \operatorname{erf}(b z) \operatorname{erf}(c z) d z \\
& -\frac{2}{\sqrt{\pi}(n+1)} \int_{0}^{\infty} z^{n+1} \exp \left[-\left(p+b^{2}\right) z^{2}\right] \operatorname{erf}(b z) d z \\
- & \frac{2}{\sqrt{\pi}(n+1)} \int_{0}^{\infty} z^{n+1} \exp \left[-\left(p+b^{2}\right) z^{2}\right] \operatorname{erf}(c z) d z,
\end{aligned}
$$

so the result follows by application of Lemma B1. The initial values given follow by equation (2.8.19.8) in Prudnikov et al. (1986, volume 2).

Lemma B3 Let

$$
K(n, p, b, c)=\int_{0}^{\infty} z^{n} \exp \left(-p z^{2}\right) \operatorname{erf}(b z) \operatorname{erf}(\mathrm{i} c z) d z
$$

for $n \geq 1, \operatorname{Re}(p)>0, \operatorname{Re}(b)>0, \operatorname{Re}(c)>0, \operatorname{Re}\left(p-c^{2}\right)>0$ and $\operatorname{Re}\left(p+b^{2}-c^{2}\right)>0$, where $\mathrm{i}=\sqrt{-1}$. Then

$$
\begin{array}{rl}
(n+1) K(n, p, b, c)=-2 p & K(n+2, p, b, c)+\frac{n \sqrt{b^{2}-c^{2}}}{\mathrm{i} \sqrt{\pi} b c} I\left(n-1, p, \sqrt{b^{2}-c^{2}}\right) \\
& -\frac{n}{\sqrt{\pi} b} I\left(n-1, p+b^{2}, \mathrm{i} c\right)-\frac{n}{\sqrt{\pi} c} I\left(n-1, p-c^{2}, b\right) \\
+ & \frac{2 p \sqrt{b^{2}-c^{2}}}{\mathrm{i} \sqrt{\pi} b c} I\left(n+1, p, \sqrt{b^{2}-c^{2}}\right)-\frac{2 p}{\sqrt{\pi} b} I\left(n+1, p+b^{2}, \mathrm{i} c\right) \\
& -\frac{2 p}{\sqrt{\pi} c} I\left(n+1, p-c^{2}, b\right)
\end{array}
$$

with the initial value

$$
K(1, p, b, c)=\frac{b I\left(0, p+b^{2}, \mathrm{i} c\right)+\mathrm{i} c I\left(0, p-c^{2}, b\right)}{\sqrt{\pi} p} .
$$

Proof: By integration by parts,

$$
\begin{aligned}
& \int_{0}^{\infty} z^{n} \exp \left(-p z^{2}\right) \operatorname{erf}(b z) \operatorname{erf}(\mathrm{i} c z) d z \\
= & {\left[z^{n} \exp \left(-p z^{2}\right) \int \operatorname{erf}(b z) \operatorname{erf}(\mathrm{i} c z) d z\right]_{0}^{\infty} } \\
& -\int_{0}^{\infty} z^{n-1}\left(n-2 p z^{2}\right) \exp \left(-p z^{2}\right)\left[\int \operatorname{erf}(b z) \operatorname{erf}(\mathrm{i} c z) d z\right] d z
\end{aligned}
$$

By equation (1.5.6.2) in Prudnikov et al. (1986, volume 2),

$$
\begin{aligned}
\int \operatorname{erf}(b z) \operatorname{erf}(\mathrm{i} c z) d z= & z \operatorname{erf}(b z) \operatorname{erf}(\mathrm{i} c z)-\frac{\sqrt{b^{2}-c^{2}}}{\mathrm{i} \sqrt{\pi} b c} \operatorname{erf}\left(\sqrt{b^{2}-c^{2}} z\right) \\
& -\frac{1}{\sqrt{\pi} b} \exp \left(-b^{2} z^{2}\right) \operatorname{erf}(\mathrm{i} c z)+\frac{1}{\sqrt{\pi} c} \exp \left(-c^{2} z^{2}\right) \operatorname{erf}(b z) .
\end{aligned}
$$


(36) follows by combining (37) and (38) and by application of Lemma B1. By integration by parts,

$$
\begin{gathered}
\int_{0}^{\infty} z \exp \left(-p z^{2}\right) \operatorname{erf}(b z) \operatorname{erf}(\mathrm{i} c z) d z \\
=\left[-\frac{1}{2 p} \exp \left(-p z^{2}\right) \int \operatorname{erf}(b z) \operatorname{erf}(\mathrm{i} c z) d z\right]_{0}^{\infty} \\
\quad-\frac{b}{\sqrt{\pi} p} \int_{0}^{\infty} \exp \left[-\left(p+b^{2}\right) z^{2}\right] \operatorname{erf}(\mathrm{i} c z) d z \\
-\frac{\mathrm{i} c}{\sqrt{\pi} p} \int_{0}^{\infty} \exp \left[\left(c^{2}-p\right) z^{2}\right] \operatorname{erf}(b z) d z,
\end{gathered}
$$

so the initial value follows by application of Lemma B1. 